\title{
Influence relative du climat et du sol sur les potentialités forestières en moyenne montagne. Exemple des sapinières à Fétuque (Festuca silvatica Vill.) dans les Vosges alsaciennes
}

\author{
M. BECKER \\ avec la collaboration technique de $\mathrm{R}$. ScHipler \\ I.N.R.A., Laboratoire de Phyto-écologie forestiere \\ Centre de Recherches forestieres de Nancy, \\ Champenoux, F 54280 Seichamps
}

\begin{abstract}
Résumé
L'étude porte sur une centaine de relevés répartis sur tout le versant alsacien des Vosges, dans des sapinières âgées (Abies alba Mill.) caractérisées par la dominance en sous-bois de la Grande Fétuque (Festuca silvatica Vill.). Chaque relevé comporte un inventaire floristique complet, des observations pédologiques (complétées par quelques analyses de sols), des données topographiques et quelques mesures dendrométriques simples (hauteur dominante et « couple de torsion» essentiellement).
\end{abstract}

L'interprétation des données s'appuie sur l'analyse factorielle des correspondances; elle montre que la végétation et, plus encore, le sol rendent assez mal compte de la variation importante de productivité observée pour le sapin dans ce contexte de moyenne montagne (500-800 $\mathrm{m}$ en moyenne).

Par contre, les données en relation avec le climat local (pluviosité et secondairement température) apparaissent beaucoup plus discriminantes. L'étude montre qu'elles peuvent être définies de façon commode à partir des habituelles variables topographiques élémentaires que sont : l'altitude, l'exposition, la pente et la pente de la direction du sommet du versant opposé (parfois appelé «masque»). L'exposition et les pentes permettent de définir un indice de climat radiatif, selon une technique rappelée en annexe. Puis une formule simple est proposée et justifiée, qui permet de calculer une «altitude compensée » en fonction de cet indice et de l'altitude vraie. C'est l'altitude compensée qui apparaît significativement corrélée à la productivité du sapin. Par ailleurs, la distance altitudinale à la ligne de crête dans la direction ouest (celle des vents pluvieux) intervient également fortement (effet de foehn). Au-dessous de $550 \mathrm{~m}$ d'altitude locale, altitude et proximité altitudinale de la crête sont toutes deux corrélées positivement à la productivité, ceci traduisant le seul effet bénéfique de la pluviosité. Au-dessus de $550 \mathrm{~m}$, la productivité croît (avec la pluviosité) si la crête est plus proche, mais elle décrô̂t (avec la température) lorsque l'on s'élève. Dans ce deuxième cas, et dans les limites observées (celles de la sapinière à fétuque), c'est l'effet positif de l'altitude qui l'emporte.

On observe également d'excellentes corrélations entre la qualité du bois (appréciée grâce à la technique du «couple de torsion ») et les mêmes variables climatiques. La densité du bois est plus forte à proximité de la ligne de crête (pluviosité plus forte); mais, d'un autre côté, elle diminue avec l'altitude compensée locale (température moyenne plus faible). 


\section{I. - Introduction}

Dans l'étage montagnard moyen des Vosges alsaciennes et lorraines, entre 500 et $800 \mathrm{~m}$ d'altitude, il est fréquent d'observer des parcelles entières de vieilles sapinières (Abies alba Mill.), dominant un tapis herbacé souvent dense constitué pour l'essentiel d'une graminée de grande taille, la Fétuque des bois ou Grande Fétuque (Festuca silvatica Vill. $=F$. altissima All.). Localement, de telles situations peuvent se rencontrer jusqu'à $250 \mathrm{~m}$ d'altitude vers le bas et $1200 \mathrm{~m}$ vers le haut. Avec des variantes, elles se rattachent principalement à l'association du Festuceto-sylvaticaeAbieti-Fagetum des phytosociologues (sous-alliance de l'Eu-Fagenion) (CARBIEner, 1963 ; LAPRAZ, 1969, 1970).

Les sols, développés sur roche-mère gréseuse ou cristalline, sont presque toujours de type sol brun acide (parfois brun ocreux) à mull acide (ou moder).

Alors que la sapinière en place se caractérise en moyenne par une productivité élevée $\left(14 \mathrm{~m}^{3} / \mathrm{ha} / \mathrm{an}\right.$ à 100 ans) (1), le forestier éprouve classiquement les pires difficultés pour régénérer ces parcelles par la voie naturelle. Ce sont ces caractéristiques assez particulières qui ont motivé l'engagement d'une étude phyto-écologique, selon une démarche désormais classique (Becker, 1969, 1979-1 ; Picard, 1979).

Les résultats de cette étude, présentés ici, sans apporter d'explications complètes aux problèmes de régénération, ont cependant permis de confirmer le rôle primordial de la fétuque elle-même, en liaison avec les propriétés physico-chimiques de l'humus, et en interaction avec les dégâts de gibier. Il en est résulté diverses études expérimentales, dont certaines ont déjà partiellement abouti, démontrant en particulier l'existence de propriétés allélopathiques chez Festuca silvatica (BECKER, BEnNET, 1980).

Mais les conclusions les plus intéressantes et les plus directement utilisables portent sur les relations entre les caractéristiques du milieu et celles du peuplement. Les caractéristiques du milieu concernent la végétation spontanée compagne du sapin, les propriétés édaphiques et le climat local (mésoclimat). Les caractéristiques du peuplement portent sur la productivité du sapin et sur la densité de son bois.

\section{II. - Déroulement des opérations et traitement des données}

\section{1. - Le recueil des données en forêt}

\subsection{1. - Prospection préliminaire}

Le choix des points d'observation ( relevés 》) devait se faire selon les critères suivants :

- présence en sous-bois d'un tapis de fétuque de densité suffisante (coefficients

(1) Bois fort tige. Il est utile de préciser qu'il s'agit de valeurs au sens des tables de production, établies pour des peuplements pleins, purs et équiennes. La production réelle récoltée est à réduire en général de 20 à $30 \%$ p. 100 , surtout si la récolte de petits bois a été f́aible ou nulle jusqu'à 40-50 ans (volume qui est intégré dans les chiffres donnés par les tables de production). 
d'abondance-dominance 4 ou 5 de la phytosociologie, c'est-à-dire ayant un recouvrement relatif supérieur à 50 p. 100);

- peuplement forestier où le sapin domine, d'âge suffisant ( $\geqslant 60$ ans);

- variabilité maximum des conditions de roche-mère, de latitude, d'altitude et de conditions topographiques (pente et exposition en particulier).

Une prospection préliminaire du versant alsacien des Vosges, entre la latitude de Phalsbourg et le Ballon d'Alsace, a permis de repérer 168 situations a priori favorables. Un choix s'est alors opéré, sur la base d'un meilleur équilibre dans la représentation des diverses variables évoquées, et ce sont finalement 103 relevés complets qui ont été retenus et faits au cours d'une deuxième phase de terrain (voir carte de la fig. 1).

\subsection{2. - Nature des relevés}

Chaque relevé comprend un inventaire floristique complet sur une surface d'environ $400 \mathrm{~m}^{2}$, selon la méthode classique de Braun-Blanquet, la prise des données concernant le milieu physique, et des mesures concernant le peuplement.

Les données physiques concernent le sol et la topographie.

Deux échantillons de sol ont été prélevés, l'un dans l'humus (horizon $A$ ou $A_{1}$ ), l'autre dans l'horizon situé au-dessous; ainsi qu'un fragment de la roche mère, pratiquement toujours accessible à moins de $70 \mathrm{~cm}$. Ramenés au laboratoire, ces échantillons ont fait l'objet d'une mesure de $\mathrm{pH}$ et de leur capacité au champ.

Les données topographiques sont : l'altitude, l'exposition z (mesure de l'azimut magnétique), la pente $\mathrm{p}$ du terrain, ainsi que la pente $\mathrm{p}^{\prime}$ de la direction du sommet du versant opposé. Les valeurs $z$, p et $\mathbf{p}^{\prime}$ permettent de définir des «indices de climat radiatif », Ir et Ig, selon une technique exposée par ailleurs (BECKER, 1979-2) et succinctement rappelée en annexe.

Les mesures concernant les peuplements sont les suivantes :

- surface terrière sur une placette circulaire de $10 \mathrm{~m}$ de rayon (ramenée ensuite à 1 'hectare) (1);

- mesure de la hauteur de deux arbres pris parmi les dominants; selon les cas, leur âge est apprécié, soit par comptage sur une souche proche, soit par l'intermédiaire du diamètre sous écorce et de la largeur moyenne des cernes, mesuréc sur une carotte de 7 à $10 \mathrm{~cm}$ extraite à la tarière de Pressler (voir fig. 2) (2);

- mesure du «couple de torsion» nécessaire à l'enfoncement de la tarière de Pressler, selon la technique de Polge et Keller (1970), comme approche de la densité du bois, qui est une de ses principales caractéristiques technologiques;

- mesure du microclimat lumineux au niveau du tapis herbacé, à l'aide d'une technique faisant appel aux photographies hémisphériques (BECKER, 1971).

(1) Compte tenu de la surface de la placette, les chiffres obtenus ont surtout une valeur indicative, permettant seulement un classement relatif.

(2) La mesure de l'âge est donc incertaine (et vraisemblablement sous-estimée). Mais elle a paru suffisante, dans un premier temps, compte tenu du nombre assez important de placettes, pour un classement relatif des divers types de milieux, bien caractérisés par ailleurs. 


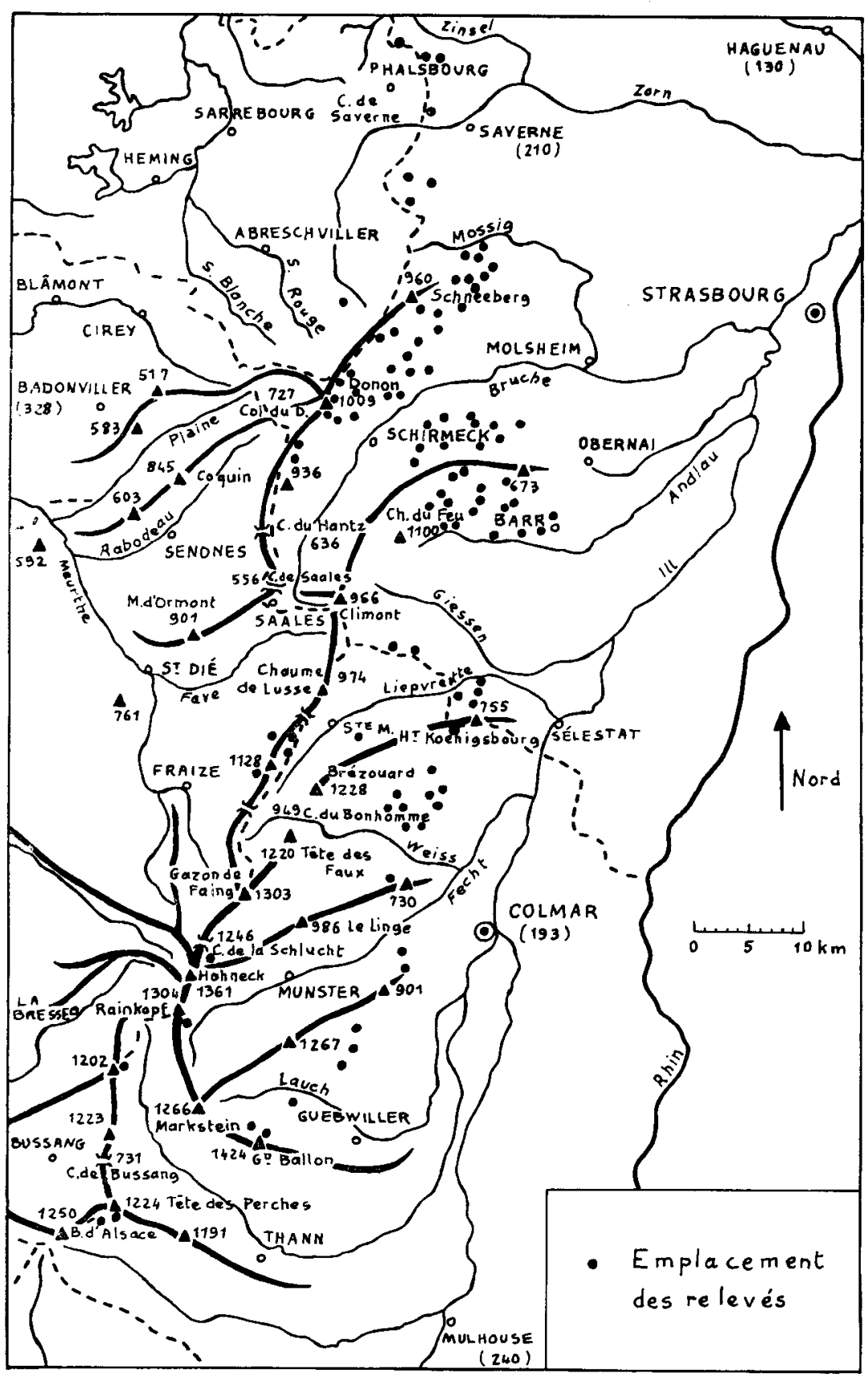

FIG. 1

Carte des Vosges et répartition des relevés sur le versant alsacien

Map of the Vosges mountains and distribution of the experiment plots on the Alsatian side 


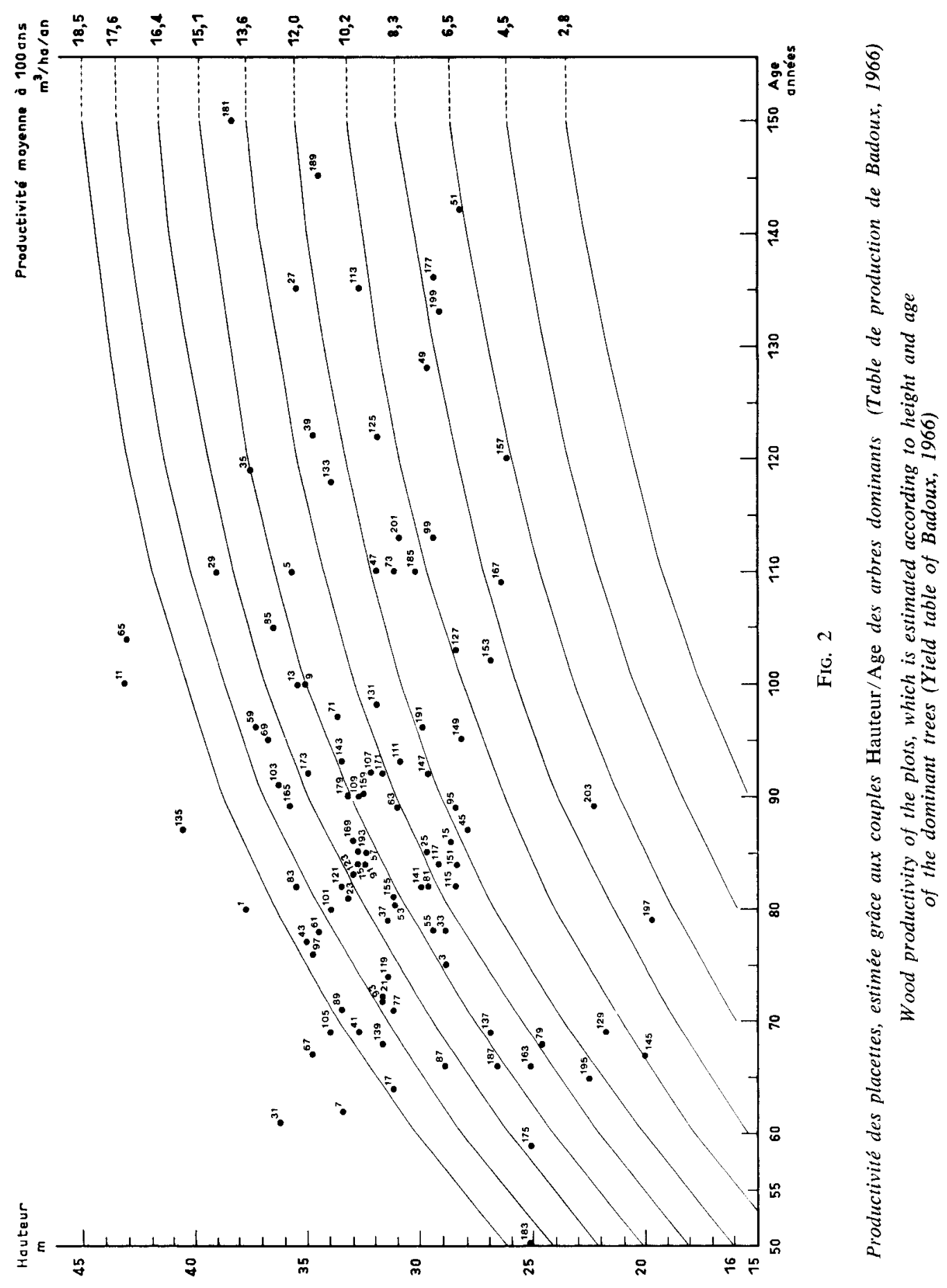




\section{2. - Le traitement des données}

La première phase de l'interprétation des données a commencé par une analyse factorielle des correspondances, d'utilisation désormais classique en phyto-écologie forestière (Juratic \& Plan, 1976 ; PiCard, 1979 ; BeCKer, 1979-1). Le programme ANALY, mis au point par Riela \& DesJeunes à la Station de Biométrie du C.N.R.F. (I.N.R.A.), a été mis en œuvre (BaCHacou, 1975). Les individus sont les relevés et les variables sont les espèces ; les données écologiques et dendrométriques, sans participer à l'analyse proprement dite, sont introduites comme variables supplémentaires facilitant interprétation des axes factoriels et un début d'explication de certaines variables dendrométriques.

Cette première interprétation a permis d'émettre des hypothèses quant au déterminisme de la variabilité importante constatée dans la productivité du sapin, mais qui s'est avérée assez médiocrement corrélée avec les critères phyto-écologiques classiques (végétation et sols). D'autres indices, d'ordre climatique, ont alors été imaginés (altitude «compensée» en particulier; voir plus loin), puis testés à l'aide du programme REPRG (régression progressive multiple) mis au point par BIENAIMÉ (Station de Biométrie du C.N.R.F.).

\section{III. - Résultats de l’analyse des correspondances}

\section{1. - Interprétation des axes factoriels}

L'analyse a porté sur les 103 relevés et les 149 espèces rencontrées. Les quatre premiers axes factoriels ont pu être interprétés, qui absorbent au total 18 p. 100 de la variation totale, successivement : 6,3 p. $100,4,5$ p. $100,3,7$ p. 100 et 3,5 p. 100 . Ces chiffres peuvent paraître faibles, mais sont dans la moyenne de ce que l'on observe lorsque toutes les espèces entrent dans l'analyse.

Les coordonnées des espèces sur l'axe 1 répartissent nettement celles-ci selon un gradient d'acidité. A une extrémité se trouvent des espèces très acidiphiles (telles que Vaccinitum myrtillus, Maianthemum bifolium, Pleurozium schreberi), puis un peu moins (Melampyrum pratense, Deschampsia flexuosa, Polytrichum formosum, Sorbus aucuparia, Luzula albida...) ; puis les espèces seulement acidoclines typiques de l'association à Grande Fétuque (Festuca silvatica, Oxalis acetosella, Athyrium filix femina, Polystichum dilatatum, Solidago virga aurea...) ; puis des espèces neutrophiles à plus ou moins large amplitude (Asperula odorata, Cardamine flexuosa, Carex silvatica, Hedera helix, Fraxinus excelsior, Geranium robertianum, Impatiens noli tangere, Lamium galeobdolon, Lysimachia nemorum, Melica uniflora, Polystichum filix mas, Senecio fuchsii, Viola silvestris, etc.) ; enfin quelques espèces considérées par ailleurs comme neutrophiles strictes ou neutro-calcaricoles (Mercurialis perennis, Stachys silvaticus, Actaea spicata, Dentaria pinnata...). Deux relevés très originaux sont caractéristiques de cette tendance extrême; il s'agit de sapinières à Dentaire qui, si elles sont courantes dans les montagnes telles que le Jura, sont tout à fait exceptionnelles, au moins dans les Vosges sur des sols non calcaires, mais à humus presque saturé (93 p. 100 , pH 5,8). 
L'interprétation de l'axe 1 est confirmée par les mesures de $\mathrm{pH}$; elles sont très significativement corrélées avec les coordonnées des relevés sur l'axe ( $r=0,44$ pour 101 échantillons). On aurait pourtant pu attendre un meilleur coefficient de corrélation ; le pH napparaît pas être un excellent indice, et le taux de saturation, s'il était plus facilement mesurable, serait sans doute bien meilleur.

L'axe 2 traduit un phénomène d’ordre climatique et phytogéographique. Il correspond à trois relevés où l'altitude, l'exposition et/ou la faible pente font que l'on passe au Melico-Ahieto-Fagetum (Lapraz, 1967), plus chaud, où l'espèce différentielle est avant tout le Chêne sessile, mais aussi le Charme, le Chèvrefeuille (Lonicera periclymenum), Holcus mollis. Les variables supplémentaires "surface terrière totale ", «microclimat lumineux ( (photographies hémisphériques), «indice de climat radiatif » (pente et exposition) confirment cette interprétation; les diverses classes de ces variables s'échelonnent en effet assez régulièrement au long de l'axe 3. L'altitude est également assez bien corrélée à cet axe : les peuplements apparaissent significativement plus denses aux altitudes fortes quaux altitudes faibles.

L'axe 3 est peu intéressant pour la définition des milieux. Il ne révèle que les espèces ayant un caractère héliophile plus ou moins marqué (Epilobium angustifolium, E. montanum, Galium rotundifolium, $G$. hercynicum, Hypericum perforatum, $H$. pulchrum, Potentilla fragariastrum, Fragaria vesca, Sarothamnus scoparius, etc.), par opposition aux espèces plutôt sciaphiles (semis d'Erable sycomore, Lamium galeobdolon, Polystichum dilatatum, etc.).

C'est l'interprétation de l'axe 4, bien que celui-ci n'absorbe que 3,5 p. 100 de la variation floristique observée, qui a été génératrice des réflexions les plus fructueuses pour la suite de l'étude. La projection des espèces sur cet axe fait ressortir, d'un côté des espèces telles que Carex silvatica, C. remota, Chrysosplenium oppositifolium, Festuca gigantea, Lysimachia nemorum, Valeriana officinalis, Polystichum spinulosum, qui ont en commun un caractère plus ou moins hygrophile, de l'autre côté des espèces, moins nombreuses, telles que Corylus avellana, Hedera helix, Ilex aquifolium, Pleurozium schreheri, Melandryum silvestre, qui, dans le contexte étudié, paraissent plutôt correspondre à des milieux plus secs. L'axe 4 semble donc être en relation avec un gradient d'humidité du milieu. Mises en corrélation avec les coordonnées des relevés sur cet axe, les valeurs de capacité au champ du sol ne donnent cependant qu'un coefficient $r=0,15$, à peine significatif au risque de 10 p. 100 . Mais ces valeurs ne traduisent pas les différences qui existent dans les apports d'eau dus à la position topographique et à la pluviosité. Nous reviendrons plus loin sur ces points.

\section{2. - Productivité et qualité du bois}

Le couple de torsion (lié à la densité du bois) avait été introduit comme variable supplémentaire. Il apparaît corrélé aux axes 3 et 4 . Pour l'axe 3, deux facteurs peuvent entrer en jeu; d'une part la densité du peuplement (traduit par la variable «surface terrière ») : la densité du bois est plus forte dans les peuplements à plus forte surface terrière; d'autre part aussi l'altitude : la densité du bois est plus forte aux altitudes élevées. Pour l'axe 4, nous avons vu qu'il semblait refléter l'alimentation en eau : les densités du bois les plus fortes sont observées dans les milieux les moins humides. Nous reviendrons en détail sur ces points. 


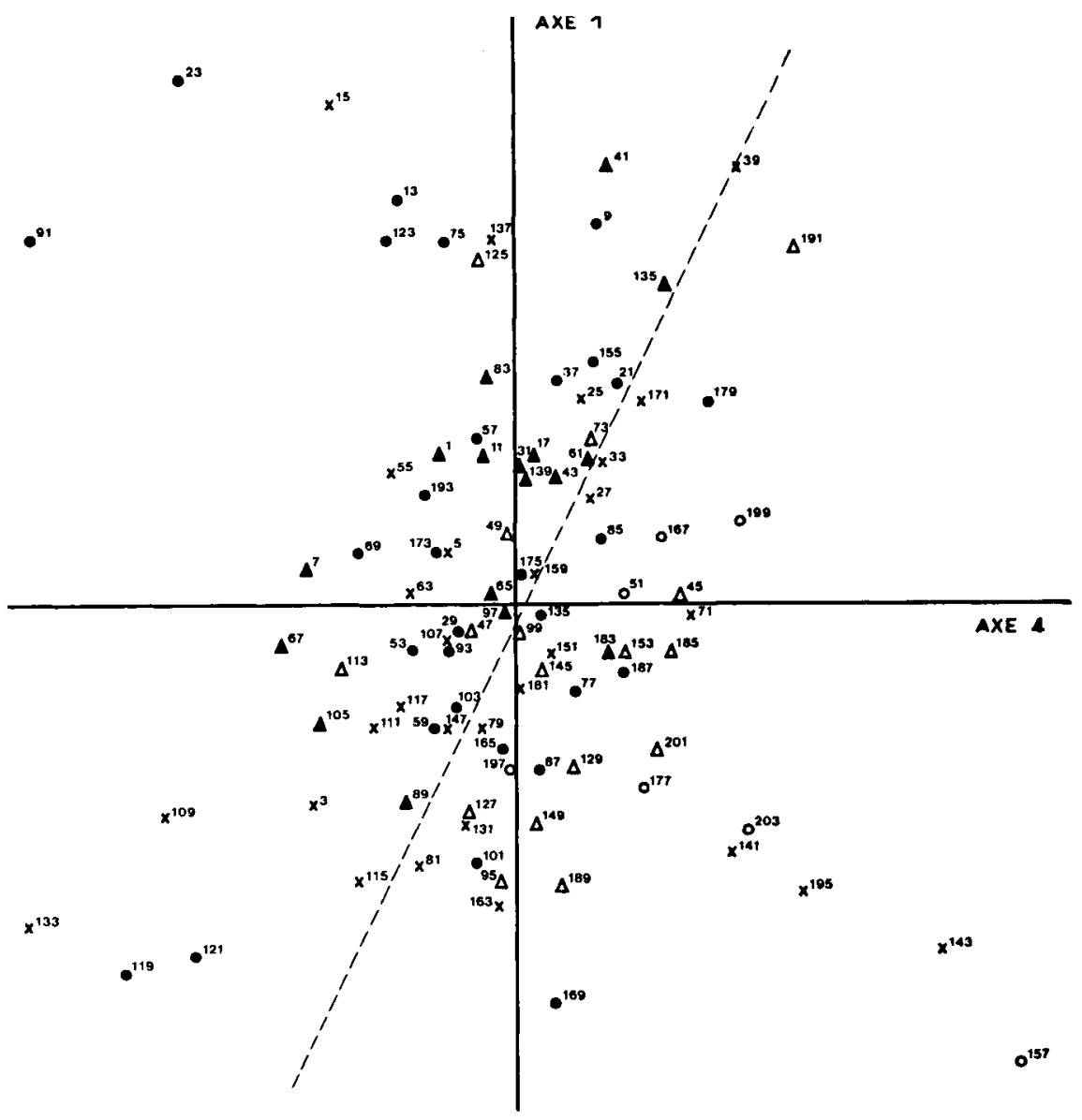

Légende:

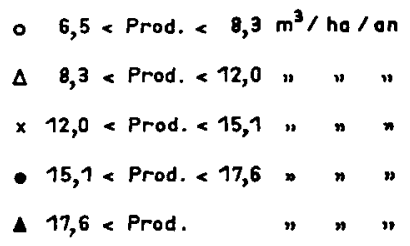

FIG. 3

Résultat de l'analyse des correspondances.

Position des relevés, classés par gammes de productivité, dans le plan factoriel 1-4 $A$ result of the analysis of correspondences. Position of the floristical "relevés» in the factor plane 1-4 and wood productivity of the corresponding plots 
Pour la productivité, nous avons utilisé les tables de production pour le sapin en Suisse, de l'Institut fédéral de Recherches forestières (Badoux, 1966) (1). Les couples hauteur-âge se sont répartis en 9 classes de productivité, variant de 7,4 à $19,5 \mathrm{~m}^{3} / \mathrm{ha} / \mathrm{an}$ depuis l'origine à 100 ans (voir fig. 1). La figure 3 indique la position de chaque relevé dans le plan factoriel 1-4, ainsi que sa fertilité estimée.

Il est assez frappant de constater, d'une part la variabilité très importante de la productivité du sapin, dautre part le peu de netteté relatif de la liaison entre productivité et composition floristique, alors que cette dernière, nous le verrons, est bien corrélée aux variations édaphiques. On observe cependant que le plan factoriel 1-4 se partage en deux zones : les productivités supérieures à la moyenne s'observent essentiellement pour les valeurs négatives sur l'axe 4, correspondant elles-mêmes aux relevés plus hygrophiles. L'influence de l'axe 1 (lié au $\mathrm{pH}$ des sols) est très discrète : Ies milieux les plus acidiphiles apparaissent même en moyenne les plus productifs.

Toutes ces observations marquent des tendances plus ou moins floues, qui vont s'éclairer dans la suite. Pour l'instant, il apparaît que les liaisons entre productivité et caractéristiques chimiques des sols sont faibles, et quelles sont plus nettes avec l'alimentation en eau; celle-ci pourrait donc bien être le facteur limitant dans le contexte étudié.

\section{3. - Elaboration du tableau floristique}

Les résultats de l'analyse des correspondances ont facilité la construction du tableau floristique ci-joint (tableau 1, voir encart). La «diagonalisation» de ce tableau a été faite avant tout en fonction du $\mathrm{pH}$, que lanalyse a reconnu comme le facteur essentiel. Toutefois, nous avons tenté de voir le parti qui pourrait être tiré de la végétation pour caractériser la fertilité des stations.

Dans ce but, parmi les 71 relevés présentés dans le tableau, nous avons séparé ceux où la productivité était supérieure à la moyenne observée $\left(14,3 \mathrm{~m}^{3} / \mathrm{ha} / \mathrm{an}\right) \mathrm{de}$ ceux où elle était inférieure. Parallèlement, ont été isolées les espèces plus spécialement fréquentes dans lun ou l'autre groupe de relevés.

On constate que la majorité des espèces, et surtout des espèces fréquentes, ne sont pas corrélées à la productivité. De part et d'autre du groupe des acidoclines, centré sur Festuca silvatica (Abies alba, Oxalis acetosella, Prenanthes purpurea...), on trouve, d'une part les acidophiles strictes (Vaccinium myrtillus, Deschampsia flexuosa, Luzula albida...), dautre part des neutro-acidoclines et des neutrophiles à plus ou moins large amplitude (Stellaria nemorum, Melica uniflora, Asperula odorata, Lamium galeobdolon, Polystichum filix mas...), certaines ayant en plus un caractère nitrophile marqué (Urtica diö̈ca, Geranium robertianum, Stachys silvaticus...).

Il est cependant possible d'isoler deux lots d'espèces ayant une fréquence d'apparition plus marquée dans l'un des deux ensembles de productivité du sapin. A l'intérieur de chaque lot, les espèces sont également classées en fonction de leur position sur l'axe 1 de l'A.F.C., c'est-à-dire en fonction du pH.

(1) D’autres tables auraient pu être utilisées, telle celle établie au Württemberg. Celle du Centre technique de l'O.N.F., pour le Jura, a paru trop tardivement pour notre étude. Mais il $\therefore$ agit seulement pour nous ici d'avoir des ordres de grandeur raisonnables. 
Si nous admettons, comme le suggère l'interprétation de l'axe factoriel $n^{\circ} 4$ (cf. § 3.1.), que la productivité est largement liée au bilan hydrique des stations, les espèces caractérisant les meilleures placettes pourraient être réparties en trois catégories :

- espèces caractérisant des sols à meilleure capacité de rétention en eau : Atrichum undulatum, Polystichum spinulosum, Ajuga reptans, Elymus europaeus, Festuca gigantea, Carex silvatica ;

- espèces liées à des possibilités locales d'alimentation en eau latérale : Moehringia trinervia, Lysimachia nemorum, Carex remota;

- espèces de sols plus désaturés, mais où la pluviosité serait, en liaison avec l'altitude, plus importante : Polytrichum formosum, Picea abies G, Dicranum scoparium, Luzula silvatica.

De la même façon, les espèces caractérisant les placettes où la productivité est inférieure à la moyenne pourraient être réparties entre les catégories suivantes :

- espèces de sols où la faiblesse de la capacité de rétention hydrique serait le facteur limitant : Ilex aquifolium, Pleurozium schreberi;

- espèces de stations où, en liaison avec une altitude trop faible, la pluviosité est insuffisante : Euphorbia amygdaloïdes, Thuidium tamariscifolium, Corylus avellana, Asperula odorata, Galium silvaticum, Melica uniflora, Paris quadrifolia, Mercurialis perennis, Geranium robertianum, Hedera helix, Melandryum silvestre, Scrofularia nodosa, Fraxinus excelsior G. Corrélativement, ces milieux sont aussi plus chauds, et les sols moins désaturés;

- espèces d'altitude élevée : Polygonatum verticillatum.

Linterprétation de ces diverses rubriques, plus ou moins hypothétique, fait largement appel aux connaissances actuelles de laautécologie des espèces citées.

\section{IV. - Propriétés physico-chimiques des sols}

Bien que les facteurs édaphiques, au vu des résultats de l'analyse générale, ne soient pas apparus comme les plus discriminants vis-à-vis de la productivité dans la gamme des stations étudiées, quelques sols ont fait l'objet de prélèvements pour analyse physico-chimique.

Les résultats analytiques sont consignés dans le tableau 2.

\section{Relevés à végétation acidophile}

- $R$ 123. Forêt de Barr (Bas-Rhin); grès; altitude : $710 \mathrm{~m}$; pente : 38 p. 100 ; exposition : NE. Différentielles : Luzula maxima, Deschampsia flexuosa, Vaccinium myrtillus, Polytrichum formosum. Productivité estimée : $16 \mathrm{~m}^{3} / \mathrm{ha} /$ an depuis l'origine à 100 ans. 


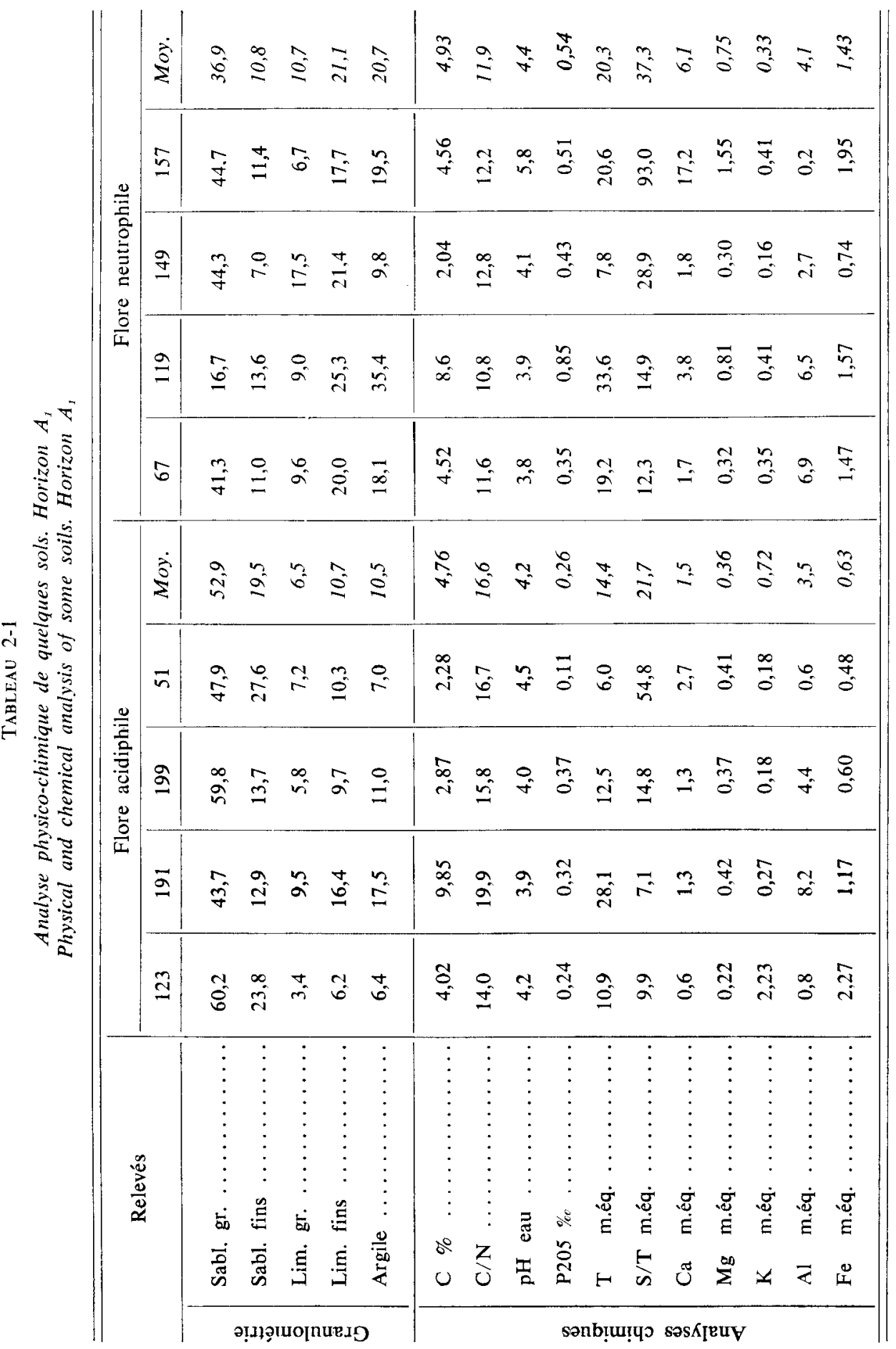




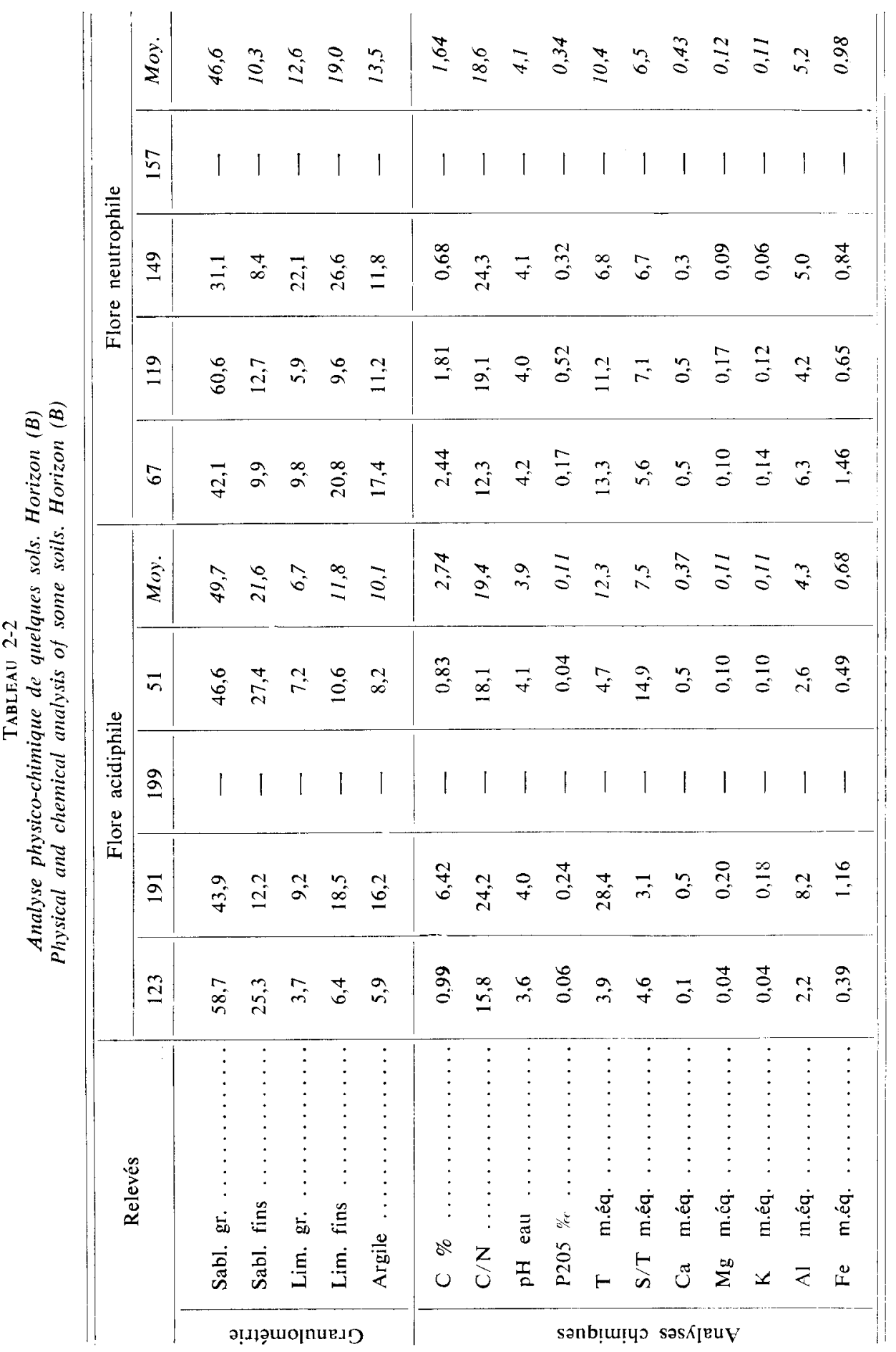


- $R$ 51. Bois de Dinsheim (Bas-Rhin) ; grès (colluvium) et granite ; altitude : $470 \mathrm{~m}$; pente : 32 p. 100 ; exposition : SSW. Différentielles : Vaccinium myrtillus, Luzula albida, L. maxima, Deschampsia flexuosa; plus des éléments neutrophiles : Geranium robertianum, Viola silvestris, Euphorbia amygdalö̈des. Productivité estimée : $7,5 \mathrm{~m}^{3} / \mathrm{ha} / \mathrm{an}$.

- $R$ 191. Forêt de la Haute-Meurthe (Vosges; un des quelques rares relevés faits sur le versant lorrain) ; granite ; altitude : $790 \mathrm{~m}$; pente : 45 p. 100 ; exposition : S. Différentielles : Deschampsia flexuosa, Luzula albida, Vaccinium myrtillus, Polytrichum formosum. Productivité estimée : $11 \mathrm{~m}^{3} / \mathrm{ha} / \mathrm{an}$.

- $R$ 199. Forêt de Rouffach (Haut-Rhin); granite; altitude : $825 \mathrm{~m}$; pente : 32 p. 100 ; exposition : NW. Différentielles : Deschampsia flexuosa, Luzula albida, Dicranum scoparium, Polytrichum formosum, Polygonatum verticillatum. Productivité estimée : $7,5 \mathrm{~m}^{3} / \mathrm{ha} / \mathrm{an}$.

\section{Relevés à végétation neutrophile}

- R67. Forêt de Lutzelhouse (Bas-Rhin); granite; altitude : $380 \mathrm{~m}$; pente : 51 p. 100 ; exposition : E. Différentielles : Carpinus betulus, Viola silvestris, Ajuga reptans, Mnium undulatum, Atrichum undulatum... Productivité estimée : $19,5 \mathrm{~m}^{3} / \mathrm{ha} / \mathrm{an}$.

- $R$ 119. Forêt de Barr (Bas-Rhin) ; granite ; altitude : $840 \mathrm{~m}$; pente : 12 p. 100 ; exposition : NW. Différentielles : Circaea lutetiana, Impatiens noli-tangere, Stachys silvatica, Elymus europaeus, Mercurialis perennis, Lamium galeobdolon... Productivité estimée : $17 \mathrm{~m}^{3} / \mathrm{ha} / \mathrm{an}$.

- $R$ 149. Forêt de Sélestat (Bas-Rhin) ; granite ; altitude : $290 \mathrm{~m}$; pente : 40 p. 100 ; exposition : NW. Différentielles : Circaea lutetiana, Impatiens nolitangere, Stachys silvaticus, Urtica diö̈ca, Geranium robertianum, Pulmonaria officinalis. Productivité estimée : $11 \mathrm{~m}^{3} / \mathrm{ha} / \mathrm{an}$.

- $R$ 157. Massif du Taennschel (Haut-Rhin); granite; altitude : $550 \mathrm{~m}$; pente : 29 p. 100 : exposition : NW. Différentielles : Dentaria pinnata, Mercurialis perennis, Lumium galeobdolon, Geranium robertianum, Vicia sepium. Productivité estimée : $7,5 \mathrm{~m}^{3} / \mathrm{ha} / \mathrm{an}$.

L'examen du tableau montre qu'il y a une bonne concordance entre composition floristique et variables édaphiques. Malgré la relative homogénéité générale des relevés prospectés, l'apparition, à côté du «noyau » central mésoacidophile (Fétuque, Oxalis...), d'éléments floristiques nettement neutrophiles correspond à des sols à texture plus fine (au moins dans l'horizon $A_{1}$ ), à rapport $C / N$ moins élevé, plus saturés en cations échangeables (sauf potassium), plus riches en phosphore. Avec le phosphore, le rapport $\mathrm{C} / \mathrm{N}$ apparaît d'ailleurs ici le critère le plus fiable. Le $\mathrm{pH}$ par contre n'est pas du tout discriminant.

C'est pourquoi d'autres échantillons de $\mathrm{A}_{1}$ ont fait l'objet de mesures du rapport $\mathrm{C} / \mathrm{N}$ (35 au total). Les moyennes obtenues, 16,3 dans les relevés à flore acidocline et 12,8 dans les relevés à flore neutrocline, sont statistiquement différentes au risque de 0,1 p. 100 . 
Les rapports avec la productivité sont beaucoup plus flous, comme précédemment entre productivité et végétation. Sur 35 échantillons, le coefficient de corrélation avec $\mathrm{C} / \mathrm{N}$ n'est que de $-0,13$ (NS) ; par ailleurs, sur 8 échantillons, les quelques valeurs marquant une tendance sont : 0,41 pour le taux d'argile (NS), 0,37 pour le potassium et 0,31 pour le phosphore. Dans ces tendances, étant donné la place de la granuIométrie, il est probable que l'on retrouve en partie la relation entre productivité et alimentation en eau (capacité de rétention des sols).

\section{V. - Déterminisme de la productivité - Climat et sol}

Nous avons déjà souligné, d’une part la relative homogénéité floristique ct édaphique des relevés prospectés, d'autre part, et au contraire, la variabilité importante observée pour la productivité du sapin; celle-ci est comprise entre 7 et $19 \mathrm{~m}^{3} / \mathrm{ha} / \mathrm{an}$, depuis l'origine à 100 ans (moyenne : 14,3; écart-type : 3,3).

L'interprétation de l'axe 4 a suggéré l'importance que pouvait avoir l'alimentation en eau sur la productivité. Or, dans les régions de montagne étudiées, la disponibilité de l'eau (pluie et évapotranspiration en particulier) est largement sous la dépendance de la topographie. Une attention toute particulière a donc été portée aux facteurs topographiques.

\section{1. - Notion d'altitude compensée}

La première variable qui vient à l'esprit est l'altitude (Oswald, 1969). Or le coefficient de corrélation entre altitude et productivité, calculé pour l'ensemble des relevés, est pratiquement nul. Afin de réduire la part de variation due à des facteurs régionaux (roche-mère et climat général en particulier), la corrélation fut ensuite calculée sur la fraction des 33 relevés situés dans le sud du domaine prospecté (au sud de la latitude de Sélestat et de la vallée de la Liepvrette; voir carte). Le coefficient de corrélation est alors de 0,13 , valeur non négligeable, mais non encore statistiquement significative.

L'idée fut alors émise que les variations d'exposition pouvaient interférer avec l'altitude brute prise en compte, au point de masquer la liaison pressentie. Nous avons donc cherché à «compenser » l'altitude mesurée en fonction non seulement de l'exposition, mais aussi de la pente.

Pour cela, nous avons utilisé des indices, mis au point précédemment (BECKER, 1979-2), qui permettent de condenser en une seule variable continue les habituelles notations de pente et d'exposition.

La suite des opérations repose sur le raisonnement suivant : comme pour la plupart des associations végétales de montagne, on peut penser que la présence du type «sapinière à fétuque» est, sur un type de sol plus ou moins constant, la résultante d'un certain nombre de paramètres responsables du climat caractérisant ce groupement végétal («climax climatique»). Parmi ces paramètres doivent logiquement figurer l'altitude, la pente et l'exposition. Nous avons donc mis en corrélation, pour 
les mêmes 33 relevés de la zone sud, puis pour les 38 relevés situés au nord de Schirmeck et de la vallée de la Bruche (voir carte), l'altitude brute Ao et lindice Ir évoqué plus haut, qui condense pente et exposition (voir fig. 4).

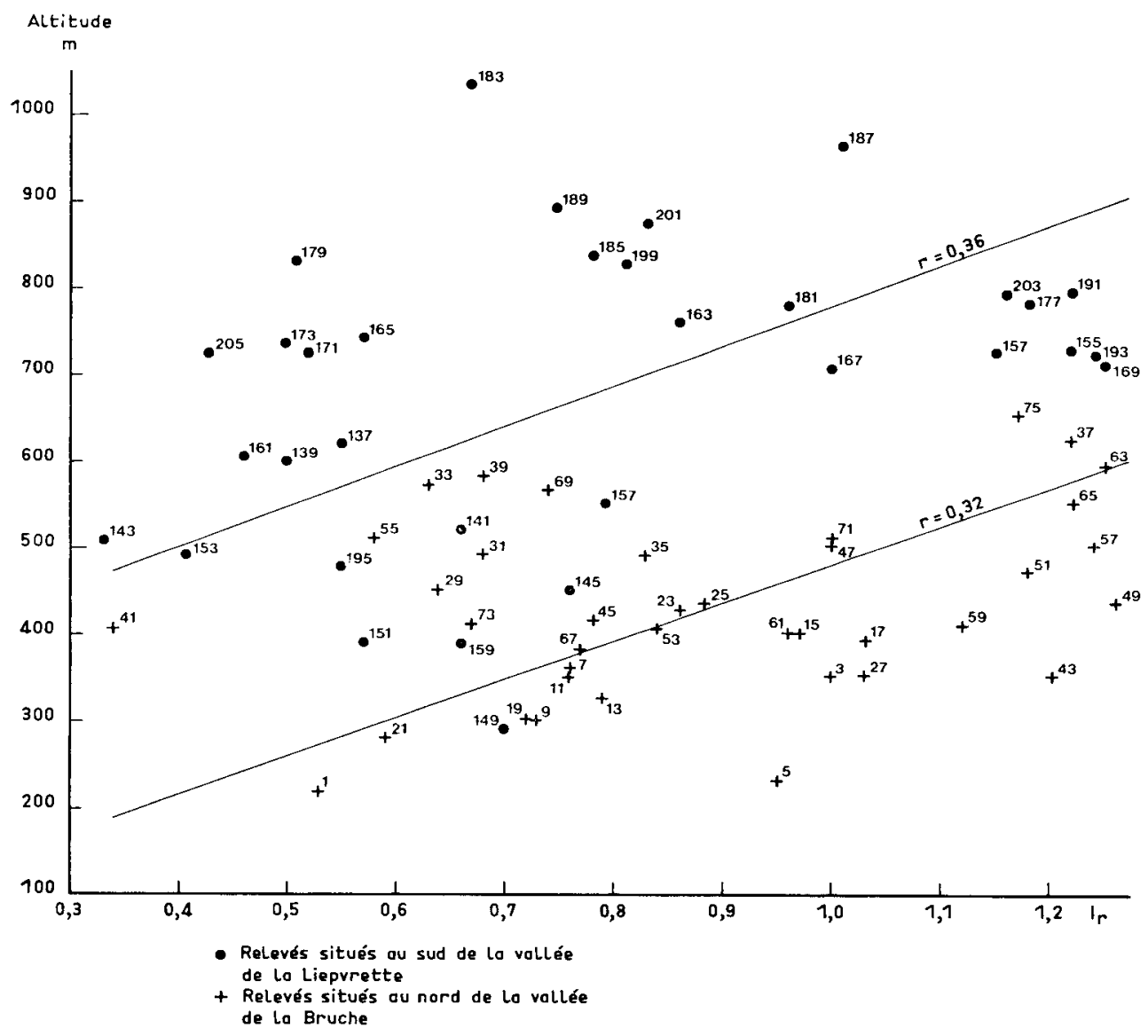

FIG. 4

Position relative des relevés en fonction de l'altitude et de lindice de climat radiatif ir combinant pente et exposition

Relative position of the experiment plots according to their altitude and to their light climate index, which combines slope and exposure

Les coefficients de corrélation obtenus sont respectivement de $0,36^{* *}$ et $0,32^{* * *}$ (1) (pour l'ensemble des relevés, on obtient seulement $0,19^{\mathrm{NS}}$ ). Qui plus est, les deux droites obtenues par ajustement des deux nuages de points sont très sensiblement parallèles. L’interprétation est la suivante : ces deux ensembles de relevés appartiennent à

(1) NS: non significatif

$*$ : significatif alu risque de $10 \mathrm{p} .100$

$*$ : significatif au risque de 5 p. 100

*:*: : significatif au risque de 1 p. 100 ou moins. 
deux zones climatiques sensiblement différentes ; mais, à l'intérieur de chacune d'elles, la sapinière se cantonne à une altitude optimale, elle-même modulée en fonction de l'exposition; quelle que soit la zone concernée, il apparaît possible de «compenser » l'altitude brute d'un lieu considéré selon la formule unique suivante :

$$
\mathrm{Ac}=\mathrm{Ao}+440(1-\mathrm{Ir})
$$

Ac : altitude compensée.

Ao : altitude brute.

Ir : indice lu sur graphique (BECKER, 1979), fonction de la pente et de l'exposition.

Ainsi, pour une pente de $30^{\prime \prime}(58$ p. 100). le passage de l'exposition plein sud à l'exposition plein nord correspond, sur le plan de la résultante climatique, à une augmentation de $430 \mathrm{~m}$ de l'altitude, ce qui est considérable.

Cette formule, mise au point pour les Vosges alsaciennes, ne devra pas être utilisée sans vérification préalable dans d'autres massifs montagneux, où les lois liant l'altitude à la température et surtout à la pluviométrie peuvent se modifier.

\section{Remarque}

Les deux droites évoquées ci-dessus, liant Ao et Ir, sont séparées par une distance altitudinale d'environ $300 \mathrm{~m}$. Or, si l'on construit (voir fig. 5) le graphique Altitude/ Pluviométrie des postes météorologiques situés à l'intérieur du domaine étudié (TroisEpis, Breitenbach, Luttenbach, Aubure, Hohwald, Rothlach, Altenberg au Sud ; Weissenberg, Saverne, Lutzelbourg, Rothau, Dabo, Glacimont au Nord), on constate que chacun de ces deux groupes de postes s'alignent également sensiblement au long de deux droites séparées par la même distance altitudinale de $300 \mathrm{~m}$. Cette observation conforte donc la précédente, et suggère qu'un autre élément important, que nous allons préciser, intervient dans le déterminisme des climats locaux (mésoclimats), en particulier de la pluviométrie locale.

\section{2. - Altitude compensée et productivité}

Pour les 33 relevés de la zone sud, le coefficient de corrélation entre altitude compensée et productivité est de $0,29^{*}$ (contre $0,13^{\mathrm{NS}}$ précédemment avec l'altitude brute). La notion d'altitude compensée confirme ainsi pleinement son intérêt.

Pour les 38 relevés de la zone nord, on obtient 0,18 , valeur non négligeable, mais non statistiquement significative. On est ainsi amené à chercher d’autres explications complémentaires d'ordre climatique.

\section{3. - Importance de la distance altitudinale de la crête}

La pluviométrie semble bien être, au vu de ces premières constatations, un élément déterminant de la productivité des stations étudiées. Or, si la pluviosité augmente bien, classiquement, avec l'altitude, il est également acquis que le versant alsacien des Vosges, à une altitude donnée, est nettement moins arrosé que le versant lorrain 
(Dion, 1972 ; Cachan, 1974). Ceci s'explique par une sorte d'effet de foehn : les vents pluvieux sont à forte dominance ouest; en montant sur le versant lorrain, l'air se détend et se refroidit, se déchargeant alors d'une grosse partie de son humidité ; une fois la crête franchie, les masses d'air redescendent, se réchauffent, et l'humidité relative diminue fortement. Ceci explique, par exemple, la «poche de Colmar», pôle de la sécheresse en France (pluviosité annuelle moyenne de $519 \mathrm{~mm}$ ).

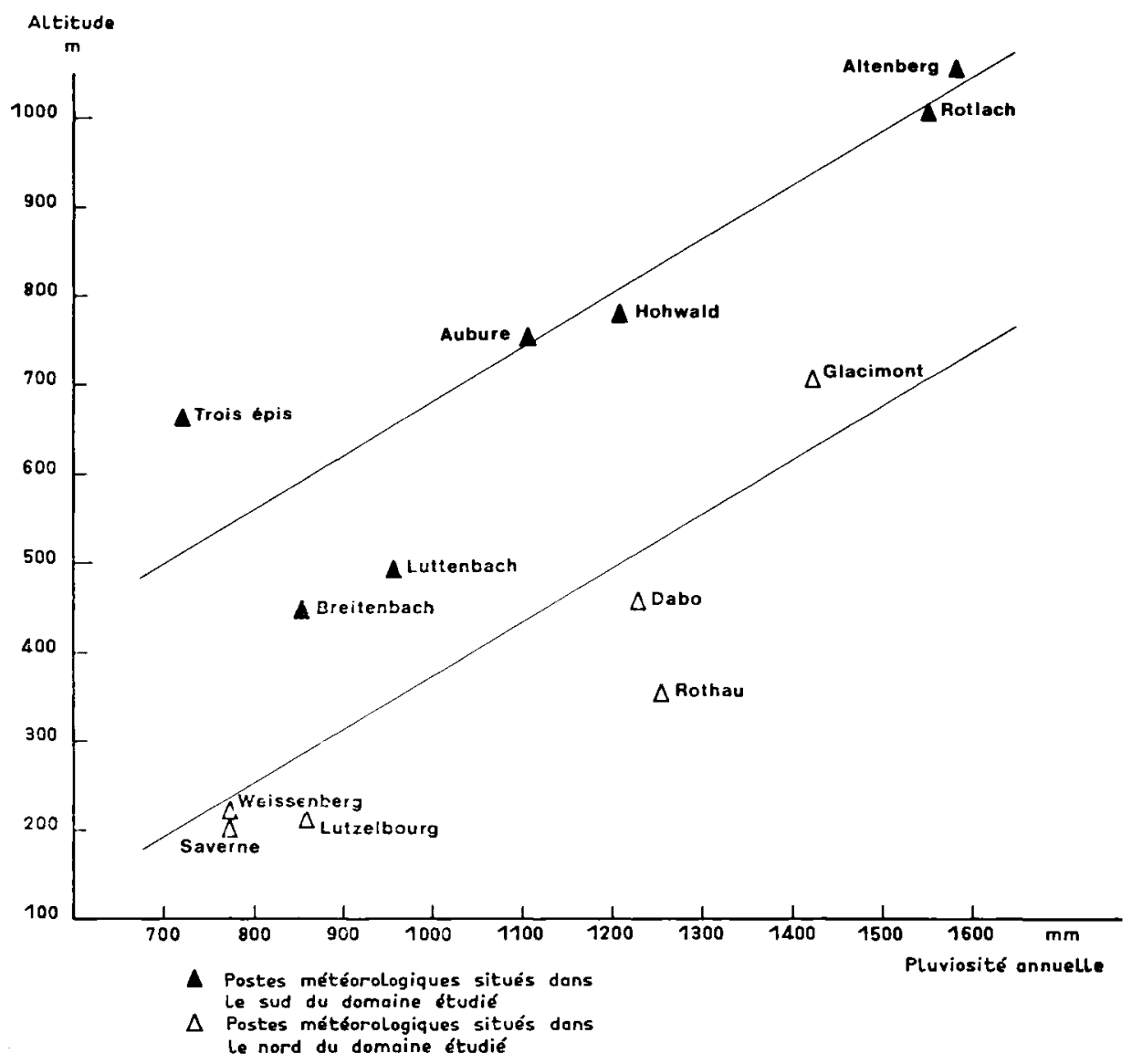

FIG. 5

Postion relative des postes mútéorologiques situés en zone forestière, en fonction de leur altitude et de la pluviosité moyenne locale

Relative position of the meteorological stations (set in forest areas) according to their altitude and to the local mean rainfall amount

Ceci nous a conduit à mesurer, pour chaque relevé, sa distance alitudinale à la crête des Vosges, dans la direction de louest, soit $\Delta_{v}$. De la même façon que précédemment, a également été calculé $\Lambda_{*}$, distance altitudinale compensée. 
Pour les relevés de la zone nord, la corrélation de la productivité est de

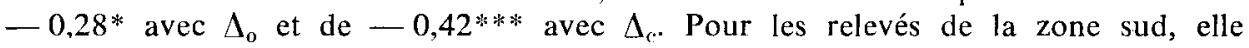
est respectivement de $-0,27$ * et $-0,38 * *$. Pour les 28 relevés de la zone intermédiaire, située entre Schirmeck et Barr (massif du Champ du Feu au sens large), pour laquelle la corrélation avec l'altitude, même compensée, est nulle, on obtient $-0,31^{*}$ (avec $\Delta_{\mathrm{c}}$ ). Enfin, en confrontant l'ensemble des 99 relevés, on trouve $-0,33^{* * *}$ (avec $\Lambda_{c}$ ).

En conséquence, dans le contexte étudié, qui est relativement peu contraignant sur le plan édaphique (nutrition minérale en particulier), la productivité du sapin apparaît fortement dépendante de la pluviosité; en effet, celle-ci croît lorsque $\Delta_{c}$ décroît, c'est-à-dire lorsque l'on se rapproche de la ligne de crête.

\section{Remarque}

Compte tenu du très faible nombre de mesures dendrométriques effectuées sur chaque relevé, les valeurs de productivité utilisées dans les calculs ci-dessus sont fatalement fort imprécises. Il y a donc toute chance que, dans la réalité, les corrélations testées soient encore plus hautement significatives. Ce point mériterait d'être ultérieurement vérifié sur la base de données dendrométriques plus fiables.

\section{4. - Analyse conjointe des divers facteurs de la productivité}

Plus l'altitude croît, plus on se rapproche de la crête... Cette lapalissade amène à se demander si altitude et distance altitudinale à la crête interviennent ou non séparément dans lexplication de la productivité du sapin. La méthode de régression progressive a donc été utilisée pour tenter d'élucider la question.

Par ailleurs, la latitude des relevés a également été introduite dans la régression, pour voir si une variation du climat général, indépendamment des variations de conditions topographiques, n'existerait pas entre la latitude de Saverne et celle de Thann.

Nous avons aussi fait intervenir la capacité au champ des horizons $A_{1}$ et (B). Celle-ci peut en effet intervenir également dans le bilan hydrique des stations.

Enfin, mais avec les 35 relevés seulement pour lesquels nous disposions de l'analyse, nous avons ajouté le rapport $\mathrm{C} / \mathrm{N}$, pour tenter de mieux faire la part des facteurs climatiques et des facteurs édaphiques dans le déterminisme de la productivité.

Latitude et altitude compensée apparaissent fortement corrélées $(r=-0,61)$. Ceci traduit seulement le fait que l'altitude générale du massif vosgien décrôit progressivement du sud vers le nord.

Pour les 98 relevés de l'étude, l'équation de régression est la suivante (1) : $\mathbf{P}=-218,0+4,31 \mathrm{~L}-0,0036 \Delta_{\mathrm{c}}+0,011 \mathrm{H} 1$

(1) Dans toutes les équations présentées, les variables apparaissent par valeur significative décroissante. 


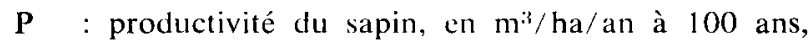

L : latitude, en grades,

$\Delta_{c}$ : distance altitudinale de la crête, en mètres,

H1 : capacité au champ, en p. 100 (en g pour $100 \mathrm{~g}$ de sol sec).

coefficient de corrélation multiple : $r=0,50^{* * * *}$.

L’introduction supplémentaire de l'altitude compensée Ac dans la régression n’améliore pas la valeur de $\mathrm{r}$. Si l'on force $\mathrm{Ac}$ à passer avant $\mathrm{L}$ dans la régression, il vient (la capacité au champ n'a pas été prise en compte dans ce calcul) :

$$
\mathrm{P}=19,8-0,0082 \Delta_{\mathrm{c}}-0,0052 \mathrm{Ac} \quad \mathrm{r}=0,42^{* * * *}
$$

Distance altitudinale à la crête et altitude interviennent donc toutes deux de façon hautement significative dans le déterminisme de la production. La capacité de rétention des sols est très secondaire : sa prise en compte n'améliore que fort peu la valeur de r $(0,50$ au lieu de 0,48$)$.

Il est important de constater que les variations d'altitude ont deux effets antagonistes : en moyenne, lorsque l'altitude croît, la distance altitudinale à la crête décroît, la pluviosité augmente, ainsi done que la productivité ; mais, par ailleurs, à position semblable par rapport à la crête $\left(\Lambda_{c}\right.$. constant), plus l'altitude est forte, plus la productivité est faible. Ce fait est d’ailleurs parfaitement traduit par le coefficient de corrélation partielle :

$$
\text { r A } \mathrm{P}, \Delta_{\mathrm{c}}=-0,27^{* * * *}
$$

Cet effet dépressif intrinsèque de l'altitude est très vraisemblablement lié à la baisse corrélative de la température moyenne (environ $0,55^{\circ} \mathrm{C}$ pour $100 \mathrm{~m}$ ).

En fait, il doit exister une altitude critique au-dessous de laquelle, à position semblable par rapport à la crête, et la température n'étant pas encore un facteur limitant, la productivité croît avec l'altitude. Nos données ont permis de vérifier cette hypothèse, et, par approches successives, d'estimer à $550 \mathrm{~m}$ cette altitude critique. En effet:

- Au-dessus de $550 \mathrm{~m}$ (62 relevés), l'équation de régression est :

$$
\mathrm{P}=-187,6-0,0064 \Lambda_{i}+3,786 \mathrm{~L} \quad \mathrm{r}=0,52 *
$$

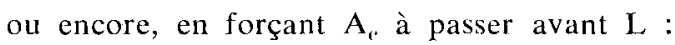

$$
P=21,93-0,0108 \Delta_{c}-0,073 A_{s} . \quad r=0,48^{* * *}
$$

Ici donc, comme dans l'équation générale, l'altitude intervient négativement, et les valeurs de $r$ sont supérieures à celles observées précédemment.

- Au-dessous de $550 \mathrm{~m}$ (37 relevés), on a :

$$
\mathrm{P}=-338,4+6,508 \mathrm{~L}+0,0068 \mathrm{~A}_{\mathrm{r}}-0,0020 \Delta_{\mathrm{r}} . \quad \mathrm{r}=0,41^{* * *}
$$

ou encore, en forçant $A_{\text {c: }}$ à passer avant $L$ :

$$
P=16,31-0,0061 \Delta_{c}+0,0015 A_{c} . \quad r=0,35^{* *}
$$

Dans ce cas, on constate donc bien que la productivité du sapin, à $\Lambda_{\text {. }}$ constant, augmente avec l'altitude. 


\section{Remarque}

Nous avons signalé la forte corrélation existant, dans le domaine étudié, entre altitude et latitude. Dans ce qui précède, on observe que la latitude a toujours un «poids» supérieur à l'altitude dans l"explication de la productivité. Sur le plan écologique, elle intègre donc «quelque chose» de plus que la seule altitude. Toutes conditions topographiques égales par ailleurs ( $A_{c}$ et $\Delta_{c}$ constantes), il pourrait s'agir d'une modification progressive du macroclimat du nord au sud de la chaîne vosgienne : diminution de la pluviosité; et/ou répartition annuelle moins favorable de cette pluviosité ? Ces hypothèses n’ont cependant pu être vérifiées.

\section{Influence du rapport carbonelazote sur la productivité}

La prise en compte sur 35 relevés du rapport $\mathrm{C} / \mathrm{N}$ fait apparaître ce dernier, de justesse au plan statistique, comme une des composantes de la productivité :

$$
\mathrm{P}=-706,6+13,42 \mathrm{~L}+0,0061 \mathrm{~A}_{\mathrm{c}}-0,2956 \mathrm{C} / \mathrm{N} \quad \mathrm{r}=0,69^{* * * *}
$$

(en l'absence de $\mathrm{C} / \mathrm{N}$, on a déjà : $\mathrm{r}=0,66$ ).

Alors que le coefficient de corrélation brut entre $P$ et $C / N$ n'était que de $-0,13^{\mathrm{NS}}$ (cf. $\$ 4$ ), le coefficient de corrélation partielle est de $-0,28$, tout juste significatif au risque de 10 p. 100.

\section{VI. - Déterminisme de la qualité du bois}

L'interprétation de l'analyse factorielle avait suggéré (cf. \$ 3.2.) l'existence de relations entre la densité du bois (appréciée par la mesure du «couple de torsion») et un certain nombre de variables écologiques et sylvicoles : surface terrière, altitude, alimentation en eau. Les valeurs mesurées, très variables, vont de 100 à $245 \mathrm{~cm} . \mathrm{kg}$ (moyenne: $157 \mathrm{~cm} . \mathrm{kg}$; écart type : 33,2).

La même technique de régression progressive multiple a été mise en œuvre sur les variables suivantes : couple de torsion, distance altitudinale compensée à la crête, altitude compensée, latitude, surface terrière, capacité au champ des horizons $A_{1}$ et $(B)$; le rapport carbone/azote a également été utilisé pour 35 relevés.

L'équation de régression obtenue (sans le rapport $\mathrm{C} / \mathrm{N}$ ) est la suivante :

$\mathrm{C}=361,3-1,325 \mathrm{~L}-0,089 \Lambda_{\mathrm{c}}-0,096 \mathrm{~A}_{\mathrm{c}}-0,262 \mathrm{HI}+0,209 \mathrm{St}$

$\mathrm{r}=0,69^{* * * *}$

C : couple de torsion, en $\mathrm{cm} . \mathrm{kg}$,

L : latitude, en grades,

$\Delta_{\text {*. }}$ : distance altitudinale compensée à la crête, en mètres,

$A_{c}$ : altitude compensée, en mètres,

H1 : capacité au champ de lhorizon Al (en p. 100),

St : surface terrière, en $\mathrm{m}^{2}$ par hectare. 
En fait, seules les trois premières variables interviennent de façon très significative (au risque de 5 p. 100); au troisième palier, la corrélation est déjà de $r=0,68 * * *$. Pour la capacité au champ et la surface terrière, on observe tout juste une tendance ; en particulier, comme il a déjà précédemment été suggéré, la densité du bois serait proportionnelle à la surface terrière.

La densité du bois de sapin est donc fortement sous dépendance climatique, et l'on retrouve, comme pour la productivité, les deux effets antagonistes de l'altitude :

- la densité augmente lorsque, à altitude constante, la ligne de crête est à une distance altitudinale plus faible (ce qui induit une pluviosité plus forte);

- mais elle diminue lorsque, à distance altitudinale constante de la ligne de crête, l'altitude est plus forte (ce qui est en relation avec une température moyenne plus faible).

On constate par ailleurs que la latitude a un «poids» encore plus fort dans la régression que précédemment pour la productivité, et qu'elle se dissocie quelque peu de l'altitude, puisque l'une et l'autre variable apparaissent significativement dans l'équation. Il est possible que ce soit la nature de la roche-mère qui entre en jeu : aux stations développées sur grès (Vosges gréseuses) correspondrait alors un bois plus tendre que pour les stations sur granite (et roches éruptives en général des Vosges cristallines). Cette conclusion est pourtant tout à fait hypothétique et mériterait d'être confirmée.

Enfin le rapport $\mathrm{C} / \mathrm{N}$, introduit dans la régression pour 35 relevés, ne «sort» pas du tout. Toutefois, compte tenu de l'observation précédente et du fait que cette variable est elle-même corrélée à la latitude et à l'altitude, il est possible qu'il intervienne de fait, comme un des paramètres traduisant les propriétés physicochimiques des sols.

\section{VII. - Conclusions}

Cette étude nous paraît remettre en cause un certain nombre de concepts sur la définition des stations forestières en moyenne montagne.

En effet, les stations sont le plus souvent caractérisées par des propriétés édaphiques particulières, un groupement végétal déterminé et, si nécessaire, par quelques paramètres en relation avec les conditions climatiques locales. Mais ce dernier aspect est généralement peu approfondi, et la position de principe est plutôt d'estimer, étant donné la difficulté de préciser les éléments du climat, que ceux-ci sont indirectement pris en compte par la végétation spontanée, qui intègre l'ensemble des paramètres stationnels.

Ceci demeure vrai à une certaine échelle de perception, et nous ne prétendons pas remettre en cause la notion fondamentale d'étage de végétation des phytogéographes. C'est d'ailleurs la réalité de cette notion qui nous a permis d'établir la formule permettant de «compenser» laltitude brute en fonction de lexposition et de la pente (cf. $\$ 5.1$.). Mais il est clairement apparu dans l'exemple traité ici, que la «sapinière à fétuque», sur sol brun acide à brun ocreux, ne pouvait être consi- 
dérée comme une station unique, étant donné la variation importante de productivité que l'on y observe ( 7 à $19 \mathrm{~m}^{3} / \mathrm{ha} /$ an à 100 ans). Cette variation n'est que très partiellement expliquée par celle des paramètres édaphiques d'une part (cf. \$ 4), et assez mal décelable à l'observation de la végétation d'autre part (cf. § 3.3.).

En plaine et en région collinéenne, les stations sont essentiellement sous déterminisme édaphique, et les relations sol-végétation, souvent étroites, rendent bien compte de la variabilité régionale.

En montagne, au contraire, le climat varie rapidement d'un point à l'autre, et devient le facteur prépondérant des variations stationnelles. Dans le cas étudié au moins, il apparaît que la végétation, toujours bien corrélée aux sols, manque alors de finesse pour caractériser des variations de productivité qu'il est important pour le forestier de prévoir. Il est donc souhaitable de prendre en compte également d`autres critères en rapport avec le climat local.

L'altitude vient naturellement à l'esprit. Mais il est nécessaire de la compenser par l'exposition (pente, orientation) au moyen, par exemple, de l'indice précédemment mis au point (Becker, 1979-2). Par ailleurs, dans les Vosges alsaciennes, où la présence d'une ligne de crête perpendiculaire à la direction des vents pluvieux dominants induit une sorte d'effet de foehn, il est également indispensable de prendre en compte la distance altitudinale à la crête.

Il apparaît qu'au-dessous de $550 \mathrm{~m}$ d'altitude, altitude et proximité altitudinale de la crête sont toutes deux corrélées positivement à la productivité, ceci traduisant le seul effet bénéfique de la pluviosité, élément essentiel de la croissance du sapin. Au-dessus de $550 \mathrm{~m}$, il convient de bien séparer ces deux variables : la productivité croît (avec la pluviométrie) si la crête est plus proche, mais elle décroît (avec la température) lorsque l'on sélève. Cette conclusion est en accord avec celle de Mettauer (1978) en milieu agricole du massif vosgien. Dans ce deuxième cas, et dans les limites observées, c'est l'effet positif de l'augmentation de l'altitude qui l'emporte.

Tous calculs faits, voici, pour différentes altitudes de la ligne de crête, la fourchette de productivité prévisible en fonction de l'altitude locale :

- crête culminant à $300 \mathrm{~m}$ : entre 14,5 et $16,8 \mathrm{~m}: 3 / \mathrm{ha} / \mathrm{an}$

- crête culminant à $550 \mathrm{~m}$ : entre 13,0 et $17,2 \mathrm{~m}^{3} / \mathrm{ha} / \mathrm{an}$

- crête culminant à $800 \mathrm{~m}$ : entre 11,4 et $16,1 \mathrm{~m}^{3} / \mathrm{ha} / \mathrm{an}$

- crête culminant à $1000 \mathrm{~m}$ : entre 10,2 et $14,6 \mathrm{~m}^{3} / \mathrm{ha} / \mathrm{an}$

- crête culminant à $1200 \mathrm{~m}$ : entre 9,0 et $13,2 \mathrm{~m}^{3} / \mathrm{ha} / \mathrm{an}$

Il resterait à quantifier la limite supérieure au-delà de laquelle l'effet dépressif de l'altitude locale devient prépondérant; mais cette limite n'est pas atteinte dans le contexte du groupement «sapinière à fétuque ».

On observe également d'excellentes corrélations entre la densité du bois (une des principales caractéristiques technologiques du bois, appréciée grâce à la mesure du «couple de torsion》) et les mêmes variables climatiques. La densité est plus forte à proximité de la ligne de crête (pluviosité plus forte) ; mais elle diminue aussi avec l'altitude locale (température moyenne plus faible).

Ce dernier résultat, dans lequel la notion d'altitude compensée s'avère également très utile, s'accorde parfaitement avec les observations de RinGaRd (1980) obtenues 
avec une espèce très différente (le mélèze), dans une autre région (Queyras et Embrunais, Hautes-Alpes) et dans une gamme daltitude supérieure (1 400 à $2200 \mathrm{~m}$ ).

Il serait souhaitable de renouveler le même type d'approche dans d'autres contextes phyto-écologiques et géographiques; en particulier pour tester le degré possible de généralisation de la formule proposée définissant l'altitude compensée ; et pour y étudier l'effet séparé de l'altitude locale et de la position des sommets avoisinants par rapport aux vents pluvieux dominants. Par ailleurs, des mesures sont en cours pour quantifier avec plus de précision la productivité de placettes de sapin dans les Vosges, afin d'améliorer les corrélations observées avec les variables topographiques.

Reçu pour publication en avril 1981.

\begin{abstract}
Summary
Relative influence of climate and soil on forest potentialities in low mountains.

Example of the white fir (Abies alba Mill.) and tall fescue (Festuca silvatica Vill.) forests in the Alsatian Vosges (N.E. France)
\end{abstract}

This study concerns about a hundred experiment plots, which are distributed along the whole Alsatian side of the Vosges mountains, in old white fir forests (Abies alba Mill.) characterized by a dense layer of tall fescue (Festuca silvatica Vill. $=F$. altissima All.). On each plot are made a complete floristical inventory ("relevé »), pedological observations (completed by some soil analysis), topographical measurements and some dendrometrical mensurations (essentially dominant height and «torsion torque», Polge, Keller, 1970).

The interpretation of the data uses the factorial analysis of correspondences; it shows that vegetation and, still more, soil, are rather badly correlated with the productivity of white fir in this region of low mountains (about $1600-2600 \mathrm{ft}$ ).

On the contrary, the data which are related to the local climate (rainfall anount and, secondarily, temperature), are much more discriminating. This study shows that they can be defined commodiously from usual topographical measures : altitude, exposure, slope and slope of the front side summit direction. Exposure and slopes allow to define a light climate index, with the aid of a technic procedure which is reminded in an annexed part. Then a simple equation is proposed and justified, which allows to calculate «corrected altitude» according to this index and to the real altitude. The corrected altitude is significantly correlated with the productivity of white fir. Otherwise the altitudinal distance to the mountain crest in the west direction (rainy winds) is strongly correlated too with productivity («foehn eftect »). Below $1800 \mathrm{ft}$ (local altitude), altitude and altitudinal distance to the crest are both positively correlated with productivity, and this expresses the only beneficial effect of rainfall amount. Above $1800 \mathrm{ft}$, productivity increases (with rainfall amount) if the crest is nearer, but it decreases (with mean temperature) if the local altitude is more elevated. For this second case, and between the studied limits (those of the fir and fescue forests) it is the positive effect of altitude which prevails.

Very good correlations are observed too between the wood technological quality (estimated with the "torsion torque» method) and the same climatical data. Wood density is ligher near the mountain crest (higher rainfall amount); but otherwise it decreases with the local corrected altitude (smaller mean temperature). 


\section{Références bibliographiques}

Bachacou J., 1975. Analyse des correspondances. Présentation du programme Analy. Document $75 / 7$ de la Station de Biométrie du C.N.R.F. (I.N.R.A.).

Badoux S., 1966. Tables de production pour le sapin en Suisse. Institut fédéral de Recherches forestières en Suisse.

BeCKer M., 1969. Le Hêtre (Fagus silvatica L.) et ses problèmes en forêt de Villers-Cotterêts (Aisne). Contribution à la mise au point d'une méthode dynamique d'étude écologique du milieu forestier. Ann. Sci. for., 26, 2, 141-182.

BECKER M., 1971. Une technique nouvelle d'utilisation des photographies hémisphériques pour la mesure du climat lumineux en forêt. Ann. Sci. for., 28, 4, 425-442.

BeCKer M., 1979-1. Une étude phyto-écologique sur les plateaux calcaires du Nord-Est (Massif de Haye - 54). Utilisation de l'analyse des correspondances dans la typologie des stations. Relations avec la productivité et la qualité du hêtre et du chêne. Ann. Sci. for., 36, 2, 93-124.

BECKER M., 1979 - 2. Indices de climat lumineux combinant pente et exposition. Bull. Ecol., 10, $2,125-137$.

BECKer M., $1979-3$. Les facteurs climatiques et la croissance du sapin dans les Vosges alsaciennes. Intérêt d'une notion nouvelle : l'altitude compensée. C.R. Acad. Agric., 65, $1307-1313$.

Becker M., Bennett P., 1980. Propriétés allélopathiques d'une graminée forestière : la Grande Fétuque (Festuca silvatica Vill.). C.R. VI Colloque international sur l'Ecologie et la Biologie des Mauvaises Herbes, Montpellier, 7-8 mai 1980, 451-460.

Cachan P., 1974. Etude bioclimatique du Massif Vosgien. Bull. E.N.S.A.I.A. Nancy, 16, $1 / 2,47$ p. +1 carte.

Carbiener R., 1963. Les sols du Massif du Hohneck; leurs rapports avec le tapis végétal. Le Hohneck, aspects physiques, biologiques et humains. Bull. Assoc. philomath. AlsaceLorraine, 7, 103-154.

Dion J., 1972. Etude fréquentielle des précipitations mensuelles du Nord-Est de la France. Rev. géogr. Est, 2/3, 175-223.

Juratic L., Plan J., 1976. Etude phyto-écologique en forêt domaniale de Grande-Chartreuse. Mémoire 3" année E.N.I.T.E.F., Nogent/Vernisson, 100 p. + annexes.

LAPraz G., 1967. Les groupements végétaux de la forêt d'Urlosenholz et de ses environs. Bull. Assoc, philomath. Alsace-Lorraine, 12, 179-221.

LAPRAZ G., 1969. Les hêtraies-sapinières à mélique et à fétuque des régions du Treiskopf, Schwarzenberg, Kagenfels et Sainte-Odile (Versant oriental des Vosges moyennes). Riviera Scientifique, 2, 31-60.

LAPraz G., 1970. Note sur les groupements forestiers et les sols sur granite de Kagenfels (Vosges moyennes). Bull. Assoc. philomath. Alsace-Lorraine, 14, 59-80.

MetTauer H., 1978. Contribution à l'étude des potentialités climatiques du Massif Vosgien. Ann. agron., 29, 6, 603-623.

Oswald H., 1969. Conditions forestières et potentialités de l'épicéa en Haute-Ardèche. Ann. Sci. for., 26, 2, 183-224.

PICARd J.F., 1979. Une méthode de définition des stations en forêt : application à la forêt domaniale de Bellême. Ann. Sci. for., 36, 3, 211-229.

Polge H., Keller R., 1970. Première appréciation de la qualité du bois en forêt par utilisation d'un torsiomètre. Ann. Sci. for., 27, 2, 197-223.

Ringard E., 1980 Etude technologique du bois de mélèze dans le Queyras et l'Embrunais. Mémoire $3^{*}$ année E.N.I.T.E.F., Nugent-sur-Vernisson, 125 p. + annexes.

Roussel L., 1952. Recherches théoriques et pratiques sur la répartition en quantité et en qualité de la lumière dans le milieu forestier. Influence de la végétation. Ann. Ec. natl. Eaux Forêts Stn Rech. Exp. for., 13, 1, 295-400. 


\section{Annexe \\ Indice de climat radiatif combinant pente et exposition}

Nous ne rappellerons ici, et très succinctement, que la notion d'indice de rayonnement direct ( $\mathbf{I r}$ ) et la démarche simplifiée permettant de calculer cet indice en fonction des conditions topographiques locales. Pour plus de détails, on se reportera à l'exposé général des techniques mises en œuvre (BECKer, 1979-2).

Il s'agit essentiellement d'une application de la notion de «cercle d'illumination totale» de Rousser. (1952). La voûte céleste peut être considérée comme une demisphère, qui se projette selon un cercle sur un plan horizontal. Le « cercle d'illumination totale» est la surface comprise entre, d'une part la projection orthogonale sur ce plan de la trajectoire apparente du soleil au solstice d'été, et, d'autre part celle correspondant au solstice d'hiver. A peu de choses près, toute cette surface (qui, en fait, se rapproche plus du croissant que du cercle) peut être considérée comme uniforme du point de vue potentialité énergétique.

En situation horizontale, nous admettons que la même quantité d'énergie serait approximativement reçue si, pendant toute la durée des jours astronomiques, le soleil demeurait immobile, dans la direction sud, à une hauteur hm au-dessus de lhorizon telle que :

$$
\mathrm{hm}=\operatorname{Arc} \cos \frac{\cos h_{1}+\cos h_{2:}}{2}
$$

$\mathrm{h}_{1}$ : hauteur du soleil au solstice dhiver.

$h_{.2}$ : hauteur du soleil au solstice d'été.

Posons :

- z : exposition d’une station donnée, mesurée par laaimut magnétique (en degrés), c'est-à-dire par l'angle que fait la direction correspondant à la ligne de plus grande pente du terrain (en observant le hat de la pente) avec le nord, dans le sens trigonométrique direct (sens inverse des aiguilles d'une montre).

- $p$ : valeur absolue de la pente du terrain, en degrés (selon la ligne de plus grande pente).

- $\mathrm{p}_{\mathrm{x}}$ : valeur algébrique de la pente dans la direction nord.

On démontre que:

$\mathrm{p}_{\mathrm{X}}=\operatorname{Arctg}(\operatorname{tg} \mathrm{p} \cdot \cos \mathrm{z})$

Si lon appelle $\mathrm{Ir}_{6}$ un indice proportionnel au rayonnement direct reçu par unité de surface horizontale dans une situation donnée, le rayonnement direct reçu par la même unité de surface parallèle au terrain est :

$\mathrm{Ir}=\mathrm{kr} . \mathrm{Ir}_{\text {, }}$

On démontre que:

$k r=\frac{\sin \left(h_{m}+p_{x}\right)}{\sin h_{m}}=\frac{\sin \left[h_{n}+\operatorname{Arctg}(\operatorname{tg} p \cdot \cos z)\right]}{\sin h_{m}}$ 

données.

Il est donc facile de calculer $\mathrm{kr}$ pour une latitude, une pente et une exposition

Pour calculer Ir, il reste à déterminer $\mathrm{Ir}_{0}$; cette valeur est directement dépendante de la forme de l'horizon apparent au lieu considéré (qui déborde plus ou moins sur la surface du ciel balayée annuellement par le soleil).

Plus précisément, $\mathrm{Ir}_{\mathrm{o}}$ est la proportion du cercle d'illumination totale occultée par la projection de l'horizon apparent sur le plan horizontal. En situation dégagée (landes, prairies, cultures...), cette valeur peut être mesurée avec précision avec la technique des photographies hémisphériques (BECKER, 1971). Mais la mise en œuvre de cette dernière est délicate, et inapplicable en forêt (horizon apparent plus ou moins masqué).

On peut tourner la difficulté par une voie graphique lorsque l'on se rapproche de la situation topographique simple suivante : vallée rectiligne, à versants symétriques et de pente plus ou moins uniforme.

Soit $\mathrm{p}$ la pente. Le plan formé par le versant sur lequel se trouve la placette étudiée recoupe la demi-sphère céleste selon un demi-cercle. Celui-ci se projette sur le plan horizontal selon une demi-ellipse, laquelle oblitère une partie du cercle d'illumination totale.

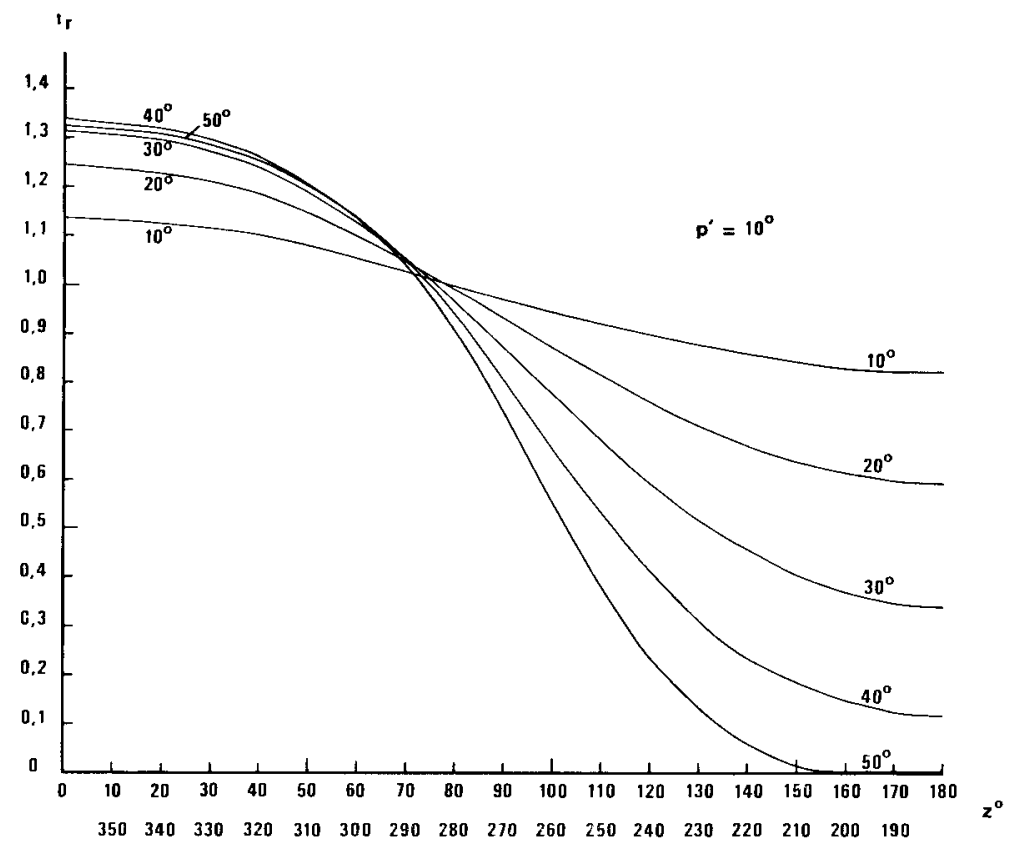

FIG. 6

Un exemple d'abaque donnant lindice de rayonnement Ir en fonction de l'exposition $\mathrm{z}$, de la pente $\mathrm{f}$ et de la pente $\mathrm{p}^{\prime}$ de la direction du faîte du versant opposé (établi pour la latitude $48^{\prime \prime} \mathrm{N}$ )

An example of the abacus which give the light climate index Ir according to exposure $\mathrm{z}$, slope $\mathrm{p}$ and slope $\mathrm{p}^{\prime}$ of the front side summit direction (for latitude $48^{\prime \prime} \mathrm{N}$ ) 
De la même façon, on peut mesurer $\mathrm{p}^{\prime}$, pente de la direction du faîte du versant opposé $\left(\mathrm{p}^{\prime} \leqslant \mathrm{p}\right)$. Le nouveau plan de pente $\mathrm{p}^{\prime}$ recoupe également la demi-sphère céleste selon un demi-cercle, qui se projette sur le plan horizontal selon une autre demi-ellipse. Celle-ci oblitère une nouvelle partie du cercle d'illumination totale.

Le calcul mathématique de la proportion du cercle d'illumination totale demeurant libre, et donc de la proportion de rayonnement direct parvenant au point considéré par rapport à une situation horizontale parfaitement dégagée, est fort complexe. Mais on peut obtenir des approximations très suffisantes par voie graphique, par planimétrage sur une maquette de taille suffisante.

On obtient ainsi, pour toutes les combinaisons des valeurs $p$, $p^{\prime}$ et $z$, les indices $\mathrm{Ir}_{0}$, correspondants, que l'on multiplie par les coefficients $\mathrm{kr}$ (obtenus par calcul) pour aboutir finalement aux indices Ir.

L'indice de rayonnement direct Ir permet donc de condenser en une seule variable continue les trois variables topographiques couramment recueillies sur le terrain, mais dont on arrivait jusqu'alors difficilement à tirer un parti totalement satisfaisant.

La figure 6 fournit un exemple des abaques que cette technique permet de construire. Il a été établi pour une latitude d'environ $48^{\circ} \mathrm{N}$ et une pente $\mathrm{p}^{\prime}=10^{\circ}$ de la direction du faîte du versant opposé.

D'autres indices ont également été proposés pour chiffrer un indice de rayonnement diffus If. Mais l'expérience a montré jusqu'ici que l'indice Ir était toujours plus discriminant. 